\title{
COMMENTARY
}

\section{Antimicrobial stewardship programs: mandatory for all ICUs}

\author{
Marin H Kollef*1 and Scott T Micek² \\ See related research by Katsios et al., http://ccforum.com/content/16/6/R216
}

\begin{abstract}
Antimicrobial stewardship involves a multifaceted approach aimed at combating the emergence of antibiotic resistance, improving patient outcomes, and controlling healthcare costs by optimizing antimicrobial use. Therefore, stewardship is of great importance and relevance in the ICU. The rapid pace of escalating antibiotic resistance and the widespread use of antibiotics in critical care require that stewardship programs be routinely employed in the ICU setting.
\end{abstract}

In this issue of Critical Care, Katsios and colleagues evaluated the impact of an antimicrobial stewardship program (ASP) introduced into a medical-surgical ICU [1]. Following implementation of the ASP there was a significant increase in the treatment of sterile site cultures and a reduction in the treatment of nonsterile site cultures representing probable colonization or contamination [1]. Additionally, utilization of the ASP was associated with increased direct antimicrobial regimen documentation, predefined antimicrobial stop dates, regimen de-escalation, and an overall reduction in cost and mean defined daily doses post ASP implementation.

Antimicrobial stewardship is an important concept that is pertinent to virtually every clinician working in the hospital. The concept involves a multifaceted approach aimed at achieving its goals of combating the emergence of resistance, improving clinical outcomes, and controlling costs by improving antimicrobial use [2]. Although progress in achieving these goals has been realized through the implementation of ASPs, there is considerable room for improvement on a global scale. Because antimicrobials are frequently prescribed in the

*Correspondence: mkollef@dom.wustl.edu

'Division of Pulmonary and Critical Care Medicine, Washington University School of Medicine, 660 South Euclid Avenue, Campus Box 8052, St Louis, MO 63110, USA Full list of author information is available at the end of the article
ICU, stewardship is particularly relevant in this setting because it provides the framework necessary to improve the quality of antimicrobial use. ASPs applied in the ICU should primarily aim to ensure that infections are treated in a timely manner with appropriate and effective regimens, that the use of unnecessary antibiotics is minimized, and that complications associated with the use of antimicrobial agents be avoided.

Given the complexity of managing infections in the critically ill it is not surprising that errors occur, resulting in either direct patient harm (for example, inappropriate initial therapy of a serious infection) or indirect patient harm (for example, prolonged unnecessary antibiotic exposure resulting in emergence of multidrug-resistant pathogens with their subsequent spread within the ICU). Nevertheless, there are some relatively simple strategies that can dramatically improve overall utilization of antibiotics as part of an ASP. First, ASPs should be multidisciplinary, taking advantage of expertise from intensivists, infectious disease specialists, microbiologists, and pharmacists [3,4]. Second, local and national protocols and guidelines should be established, with input from all stakeholders, providing basic concepts and practices that will facilitate the implementation and success of ASPs in the ICU, as well as throughout the hospital [5]. Third, the impact of the ASP should be regularly evaluated to determine its overall effectiveness and need for updating $[5,6]$. Lastly, ASPs will be most effective when they are integrated into an overall program aimed at reducing the presence of antibiotic resistance and its impact on patient outcomes, to include the use of effective infection control practices $[7,8]$.

The global spread of antimicrobial resistance has become a pressing problem, with a focus on the ICU due to the increasing administration of ineffective antimicrobial regimens associated with greater morbidity and mortality $[9,10]$. There should no longer be any debate on the need for ASPs - the debate should now center on how to most effectively employ stewardship programs, not just in the ICU setting but throughout the hospital and even in the community setting [11]. Appropriately designed and implemented ASPs can reduce the burden 
of antimicrobial resistance and reduce healthcare costs by decreasing antibiotic expenditures, lengths of stay, and the need for additional diagnostic testing $[4,5,12]$. However, additional research is urgently needed to develop alternative more effective approaches to the management of infections that will promote the principles of antimicrobial stewardship.

Advances in biomarkers such as procalcitonin and optimization of their utilization is needed to determine whether every patient in the ICU with clinical signs suggesting the presence of infection (for example, fever and/or leukocytosis) needs to be treated with antibiotics; and if so, for how long [13]. Moreover, the widespread use of broad-spectrum antibiotics in the ICU supports the need to develop rapid diagnostics aimed at determining which antibiotics should be prescribed in order to ensure that appropriate therapy is being administered and that unnecessary therapy is avoided [14]. The challenge will be to develop such assays in a way that will demonstrate their clinical utility and cost-effectiveness. There is also a critical need for new antimicrobial agents targeting multidrug-resistant pathogens [15]. Lastly, we need to acquire novel infection prevention strategies. These could potentially include vaccines and monoclonal antibodies targeting the prevention of infections caused by antibiotic-resistant pathogens (Pseudomonas aeruginosa, Acinetobacter species and methicillin-resistant Staphylococcus aureus).

In summary, there can be little debate that antimicrobial resistance has emerged as one of the most important problems impacting the care and outcomes of patients in ICUs. ASPs should be employed routinely in order to maximize the effectiveness of antibiotic therapy, not only for the infected patient but for all patients in the ICU environment. The ultimate rationale or justification for the use of ASPs in the ICU is that antibiotic therapy not only impacts the treated patient but also those patients in the surrounding environment by promoting the development of resistance.

Abbreviations

ASP, antimicrobial stewardship program.

\section{Competing interests}

MHK's efforts are supported by the Barnes-Jewish Hospital Foundation. STM declares that he has no competing interests.

\section{Author details}

'Division of Pulmonary and Critical Care Medicine, Washington University School of Medicine, 660 South Euclid Avenue, Campus Box 8052, St Louis, MO 63110, USA. 2Pharmacy Department, Barnes-Jewish Hospital, 1 Barnes-Jewish Hospital Plaza, St Louis, MO 63110, USA.

Published: 22 November 2012

\section{References}

1. Katsios CM, Burry L, Nelson S, Bell CM, Jivraj T, Lapinsky SE, Wax RS, Christian M, Mehta S, Morris AM: An antimicrobial stewardship program improves antimicrobial treatment by culture site and the quality of antimicrobial prescribing in critically ill patients. Crit Care 2012, 16:R216.

2. Lawrence $\mathrm{KL}$, Kollef MH: Antimicrobial stewardship in the intensive care unit: advances and obstacles. Am J Respir Crit Care Med 2009, 179:434-438

3. Micek ST, Ward S, Fraser VJ, Kollef MH: A randomized controlled trial of an antibiotic discontinuation policy for clinically suspected ventilatorassociated pneumonia. Chest 2004, 125:1791-1799.

4. Arnold HM, Micek ST, Skrupky LP, Kollef MH: Antibiotic stewardship in the intensive care unit. Semin Respir Crit Care Med 2011, 32:215-227.

5. Society for Healthcare Epidemiology of America; Infectious Diseases Society of America; Pediatric Infectious Diseases Society: Policy statement on antimicrobial stewardship by the Society for Healthcare Epidemiology of America (SHEA), the Infectious Diseases Society of America (IDSA), and the Pediatric Infectious Diseases Society (PIDS). Infect Control Hosp Epidemiol 2012, 33:322-327.

6. Griffith $M$, Postelnick M, Scheetz M: Antimicrobial stewardship programs: methods of operation and suggested outcomes. Expert Rev Anti Infect Ther 2012, 10:63-73.

7. Chalfine A, Kitzis MD, Bezie Y, Benali A, Perniceni L, Nguyen JC, Dumay MF,Gonot J, Rejasse G, Goldstein F, Carlet J, Misset B: Ten-year decrease of acquired methicillin-resistant Staphylococcus aureus (MRSA) bacteremia at a single institution: the result of a multifaceted program combining crosstransmission prevention and antimicrobial stewardship. Antimicrob Resist Infect Control 2012, 1:18.

8. Apisarnthanarak A, Pinitchai U, Thongphubeth K, Yuekyen C, Warren DK, Fraser VJ; Thammasat University Pandrug-Resistant Acinetobacter baumannii Control Group: A multifaceted intervention to reduce pandrug-resistant Acinetobacter baumannii colonization and infection in 3 intensive care units in a Thai tertiary care center: a 3-year study. Clin Infect Dis 2008, 47:760-767.

9. Chung DR, Song JH, Kim SH, Thamlikitkul V, Huang SG, Wang H, So TM, Yasin RM, Hsueh PR, Carlos CC, Hsu LY, Buntaran L, Lalitha MK, Kim MJ, Choi JY, Kim SI, Ko KS, Kang Cl, Peck KR; Asian Network for Surveillance of Resistant Pathogens Study Group: High prevalence of multidrug-resistant nonfermenters in hospital-acquired pneumonia in Asia. Am J Respir Crit Care Med 2011, 184:1409-1417.

10. Morata L, Cobos-Trigueros N, Martínez JA, Soriano A, Almela M, Marco F, Sterzik H, Núñez R, Hernández C, Mensa J: Influence of multidrug resistance and appropriate empirical therapy on the 30-day mortality rate of Pseudomonas aeruginosa bacteremia. Antimicrob Agents Chemother 2012 56:4833-4837.

11. George P, Morris AM: Pro/con debate: Should antimicrobial stewardship programs be adopted universally in the intensive care unit? Crit Care 2010, 14:205.

12. Nowak MA, Nelson RE, Breidenbach JL, Thompson PA, Carson PJ: Clinical and economic outcomes of a prospective antimicrobial stewardship program. Am J Health Syst Pharm 2012, 69:1500-1508.

13. Schuetz P, Briel M, Christ-Crain M, Stolz D, Bouadma L, Wolff M, Luyt CE, Chastre J, Tubach F, Kristoffersen KB, Wei L, Burkhardt O, Welte T, Schroeder S, Nobre V, Tamm M, Bhatnagar N, Bucher HC, Mueller B: Procalcitonin to guide initiation and duration of antibiotic treatment in acute respiratory infections: an individual patient data meta-analysis. Clin Infect Dis 2012, 55:651-662.

14. Lodes U, Bohmeier B, Lippert H, König B, Meyer F: PCR-based rapid sepsis diagnosis effectively guides clinical treatment in patients with new onset of SIRS. Langenbecks Arch Surg 2012, 397:447-455.

15. Coates AR, Halls G: Antibiotics in phase II and III clinical trials. Handb Exp Pharmacol 2012, 211:167-183.

doi:10.1186/cc11853

Cite this article as: Kollef MH, Micek ST: Antimicrobial stewardship

programs: mandatory for all ICUs. Critical Care 2012, 16:179. 